九州大学学術情報リポジトリ

Kyushu University Institutional Repository

RANTES has a potential to play a neuroprotective role in an autocrine/paracrine manner after ischemic stroke

砥上，妃美子

https://doi.org/10.15017/1441124

出版情報: 九州大学，2013，博士（医学），課程博士 バージョン：

権利関係 : 全文ファイル公表済 


\title{
RANTES has a potential to play a neuroprotective role in an autocrine/paracrine manner after ischemic stroke
}

\author{
Himiko Tokami ${ }^{a}$, Tetsuro Ago ${ }^{a, c, \star, \pi}$, Hiroshi Sugimoria ${ }^{a}$ Junya Kurodaa, \\ Hideto Awano ${ }^{d}$, Kazuo Suzukid ${ }^{d}$ Yutaka Kiyoharab ${ }^{b}$, Masahiro Kamouchia, \\ Takanari Kitazono ${ }^{\text {; }}$ for the REBIOS Investigators
}

${ }^{\mathrm{a} D e p a r t m e n t}$ of Clinical Science and Medicine, ${ }^{\mathrm{b}}$ Environmental Medicine \& 'Innovation Center for Medical Redox Navigation, Graduate School of Medical Sciences, Kyushu University, ${ }^{\mathrm{d} M o l e c u e n c e}$

* These authors contributed equally to this work.

ף Correspondence: Tetsuro Ago, MD, PhD

Department of Medicine and Clinical Science,

Graduate School of Medical Sciences, Kyushu University,

Fukuoka 812-8582, Japan

Phone: +81-92-642-5256, Fax: +81-92-642-5271

E-mail: agou@intmed2.med.kyushu-u.ac.jp 


\section{Abstract}

Regulated upon Activation, Normal T-cell Expressed, and Secreted (RANTES) is a well-known pro-inflammatory chemokine and its role in ischemic stroke remains controversial. We examined the significance of RANTES in ischemic stroke and aimed to elucidate the direct effect of RANTES on neurons. Plasma concentrations of major C-C chemokines, including RANTES, and neurotrophic factors were examined in 171 ischemic stroke patients and age- and gendermatched healthy subjects. Plasma concentrations of RANTES at day 0 were significantly elevated in stroke patients, compared with controls, and were highly correlated with those of BDNF, EGF, and VEGF. In a mouse middle cerebral artery occlusion model (MCAO), plasma RANTES was significantly elevated and the expression of RANTES was markedly upregulated in neurons particularly in peri-infarct areas. The expression of CCR3 and CCR5, receptors for RANTES, was also induced in neurons, while another receptor, CCR1, was observed in vascular cells, in peri-infarct areas after MCAO. We examined the effects of RANTES on differentiated PC12 cells, a model of neuronal cells. Treatment with RANTES induced the activation of Akt and Erk1/2, and attenuated the cleavage of caspase-3 in the cells. RANTES increased the expression of BDNF, EGF, and VEGF in the cells. Moreover, RANTES maintained the number of cells under serum free conditions. The RANTES-mediated upregulation of neurotrophic factors and cell survival were significantly attenuated by the inhibition of Akt or Erk1/2. Taken together, RANTES is an interesting chemokine that is produced 
from neurons after ischemic stroke and has the potential to protect neurons directly or indirectly through the production of neurotrophic factors in peri-infarct areas.

\section{Keywords}

RANTES, chemokine receptors, neurotrophic factors, ischemic stroke, blood biomarker
Abbreviations
BDNF, brain-derived neurotrophic factor; CBF, cerebral blood flow;
DAPI, 4',6-diamidino-2-phenylindole; EGF, epidermal growth factor;
GPCR, G protein-coupled receptors; MCAO, middle cerebral artery occlusion;
NGF, nerve growth factor; RANTES, Regulated upon Activation, Normal T-cell
Expressed, and Secreted; VEGF, vascular endothelial growth factor 


\section{Introduction}

Chemokines are a family of proteins secreted by cells that induce chemotaxis in nearby responsive cells. They are classified largely into 4 groups, i.e. C, C-C, CXC, and $\mathrm{CX}_{3} \mathrm{C}$ chemokines, based on their structure (Cyster, 1999; Murdoch and Finn, 2000; Murphy et al., 2000; Zlotnik and Yoshie, 2000). Some chemokines are considered pro-inflammatory and are induced during an immune response to recruit immunological cells, while others are involved in controlling the migration of cells during normal processes of tissue development or maintenance (Cyster, 1999; Murphy et al., 2000; Zlotnik and Yoshie, 2000). Chemokines exert their biological effects through $\mathrm{G}$ protein-coupled receptors (GPCR) that are selectively expressed on the surfaces of their target cells (Cyster, 1999; Murdoch and Finn, 2000; Murphy et al., 2000).

RANTES (Regulated upon Activation, Normal T-cell Expressed, and Secreted), also known as CCL5, is one of the C-C chemokines and plays an active role in recruiting leukocytes, T cells, eosinophils and basophils, into inflammatory sites, thereby functioning as a pro-inflammatory molecule (Maghazachi et al., 1996). The effects of RANTES are exerted through three different types of GPCR, i.e. CCR1, CCR3 and CCR5 (Daugherty et al., 1996; Ignatov et al., 2006; Proudfoot et al., 2001; Slimani et al., 2003; Struyf et al., 2001).

In experimental stroke models, it has been reported that infarct volume is significantly reduced in RANTES deficient mice (Terao et al., 2008). Because 
RANTES is a representative pro-inflammatory chemokine and inflammation is a detrimental factor of ischemic stroke, the results from RANTES deficient mice seem to be plausible. On the other hand, it has been recently reported that infarct volume is rather enlarged in mice with deletion of CCR5 (Sorce et al., 2010). Although these data highly suggest an important role of RANTES in ischemic stroke in mice, it is unclear why infarct volume is enlarged in CCR5 knockout mice and whether RANTES plays a significant role in human ischemic stroke.

In the present study, we examined the clinical significance of RANTES in human acute ischemic stroke. Plasma RANTES was elevated at day 0 after stroke onset and was highly associated with brain-derived neurotrophic factor (BDNF), epidermal growth factor (EGF), and vascular endothelial growth factor (VEGF), major neuroprotective molecules, suggesting a neuroprotective potential of RANTES in ischemic stroke. We also examined whether RANTES was produced and could function within the brain in the acute phase of ischemic stroke, and whether the chemokine could have a neuroprotective potential using a mouse stroke model and cultured neuronal cells. 


\section{Results}

\section{1. Plasma RANTES was significantly elevated after ischemic stroke in humans and highly correlated with neurotrophic factors}

In our blood biomarker study for acute ischemic stroke in humans, we found that plasma concentration of RANTES was much higher than that of other major C-C chemokines that we measured in this study by $50 \sim 100$-fold at baseline and was significantly elevated at day 0 after onset when compared with healthy controls (Figure 1A). Other major C-C chemokines, such as MCP-1 (CCL2) (Figure 1B), MIP-1 $\alpha$ (CCL3) (Figure 1C) and MIP-1 $\beta$ (CCL4) (Figure 1D), were not significantly altered at day 0 , suggesting that the elevation in plasma was a specific phenomenon for RANTES, and thus it may play significant roles after ischemic stroke in humans. The increase in RANTES lasted at least until day 14 after onset, and then decreased towards control levels (Figure 1E).

We also found that plasma RANTES highly correlated with BDNF (Figure 1F: $r=0.85, p<0.001$ ), EGF (Figure 1G: $r=0.80, p<0.001)$ and VEGF (Figure 1H: $r=0.34, p<0.001)$, major neuroprotective molecules, in stroke patients. These findings suggested a neuroprotective potential of RANTES in the brain after ischemic stroke.

\section{2. RANTES was expressed in neurons predominantly in the peri-infarct} area after MCAO

We next examined plasma concentration of RANTES in mice subjected to an 
MCAO stroke model (Figure 2A, B). Plasma RANTES levels were significantly higher in MCAO mice $(\mathrm{N}=8)$ than sham-operated mice $(\mathrm{N}=6)$ at 24 hours after MCAO $(p<0.05)($ Figure $2 \mathbf{C})$. In order to elucidate the origin of the increased plasma RANTES after MCAO, we examined mRNA expression levels of RANTES in several organs, such as the brain, liver, and bone marrow, by quantitative PCR. As shown in Figure 2D, the expression of RANTES was significantly elevated in the ischemic hemisphere of MCAO mice, compared to sham-operated mice. However, the expression of RANTES in the liver and bone marrow was very marginal in sham-operated and MCAO mice (data not shown).

We further examined the cell type in the brain that could produce RANTES after MCAO by immunohistochemistry. RANTES was strongly expressed in neuron-like cells particularly in peri-infarct areas at day 1 after MCAO when inflammatory cells did not yet infiltrate into the infarct area (Figure 2E, F). In contrast, the expression of RANTES remained at very low levels in the contralateral hemisphere (Figure $\mathbf{2 G}$ ) and in the brain of sham-operated mice (Figure 2H). We confirmed by immunofluorescent double labeling that RANTES was expressed in NeuN-positive neurons in peri-infarct areas at day 1 after MCAO (Figure 2I).

\section{3. Expression of receptors for RANTES in the brain after MCAO}

We next examined the expression of three known receptors for RANTES, i.e. CCR1, CCR3 and CCR5, in the brain after MCAO. Immunohistochemistry 
demonstrated that all these receptors were hardly expressed in the brain in sham-operated mice (Figures 3A-C). In contrast, the expression of CCR1 was induced in vascular cells (Figure 3D), while that of CCR3 (Figure 3E) and CCR5 (Figure 3F) was markedly induced in neurons in peri-infarct areas at day 1 after MCAO. The expression levels of CCR 3 and CCR5 were much higher in the ischemic hemisphere (Figures $3 \mathrm{E}$ and $3 \mathrm{~F}$ ) than in the non-ischemic contralateral side (Figures $\mathbf{3 H}$ and $3 \mathrm{I}$ ). We further confirmed by immunofluorescent double labeling that CCR1 was expressed in lectin-positive endothelial cells (Figure 3J), while CCR3 (Figure 3K) and CCR5 (Figure 3L) were in NeuN-positive neurons. These findings suggested that RANTES was produced from neurons and could act in an autocrine/paracrine manner in peri-infarct areas after ischemic stroke.

\section{4. Effects of RANTES on neuronal cells}

In order to examine the effects of RANTES on neurons, we employed nerve growth factor (NGF)-treated PC12 cells, which are known to differentiate into neuronal cells in response to NGF and are commonly used as a neuronal cell model (Ishitsuka et al., 2012; Song and Yoo, 2011). Treatment with RANTES induced the phosphorylation (=activation) of Akt (Figure 4A) and Erk1/2 (Figure 4B), both pro-survival molecules for neurons, and the phosphorylation (=inactivation) of GSK3 $\beta$ (Figure 4C), and attenuated the cleavage of caspase-3, a crucial inducer of apoptosis, in neuronal cells (Figure 4D). 


\section{5. RANTES increased gene expression of neurotrophic factors in} differentiated PC12 cells

We further examined the effect of RANTES on the gene expression of neurotrophic factors in NGF-treated PC12 cells. Treatment with RANTES significantly increased the expression of BDNF (Figure 5A), EGF (Figure 5B), and VEGF (Figure 5C) in the cells. The RANTES-induced upregulation of these neurotrophic molecules was significantly attenuated by the treatment with either U0126 or LY29002, an upstream inhibitor of Erk1/2 or Akt (Figures 5D-F).

\section{6. RANTES induced survival of differentiated PC12 cells}

We finally tested the pro-survival effect of RANTES on NGF-treated PC12 cells. Treatment of RANTES at concentrations of $50 \mathrm{ng} / \mathrm{mL}$ and $500 \mathrm{ng} / \mathrm{mL}$ induced survival of cells under serum free conditions (Figure 6A). The addition of either U0126 or LY294002 abolished the RANTES-mediated pro-survival effect in the cells (Figure 6B). 


\section{Discussion}

In the present study, we demonstrated that RANTES was elevated in plasma after ischemic stroke in humans and mice, and that it was produced in the brain, particularly from neurons in peri-infarct areas, in an experimental stroke model. Although RANTES is a well-known pro-inflammatory chemokine that recruits white blood cells into infarct areas (Montecucco et al., 2012) and thus has been believed to exaggerate brain infarction (Terao et al., 2008; Yilmaz and Granger, 2010), the present study suggests that RANTES produced from neurons in response to ischemia has the potential to protect neurons through receptors expressed specifically in neurons after ischemic stroke.

\section{1. Is increased plasma RANTES after ischemic stroke derived from the}

\section{brain?}

We demonstrated that RANTES was elevated in plasma after ischemic stroke in humans (Mirabelli-Badenier et al., 2011) and mice. Because elevated RANTES tended to decrease towards control levels in the chronic phase, it can be thought that the elevation of RANTES in the acute phase did not simply reflect the background characteristics of patients predisposed to brain infarction, but was induced after stroke onset. Although we examined plasma concentrations of more than 100 proteins after ischemic stroke in humans, there were only several molecules, such as BDNF, EGF and VEGF, that were significantly increased at day 0 after ischemic stroke. A common characteristic of these molecules is that 
they can be produced in the brain and have a neuroprotective role (Peng et al., 1998; Sun et al., 2003; Wu, 2005; Zhang and Pardridge, 2001). Consistently, the expression of RANTES and its receptors, CCR3 and CCR5, were markedly induced in neurons predominantly in peri-infarct areas within a day after MCAO when inflammatory cells do not yet infiltrate into infarct areas, indicating that RANTES can function in an autocrine/paracrine manner in the brain in the acute phase of ischemic stroke. In this context, we speculated that the rapid increase in plasma RANTES may be caused in part by its increased production in the brain, because the expression of RANTES in other organs we tested, such as the bone marrow and liver, was not increased after MCAO in contrast to that in the brain. However, we cannot completely exclude the possibility that peripheral white blood cells produced RANTES into the plasma in response to brain ischemia. It has been reported that plasma RANTES levels are increased in acute coronary syndrome (Nomura et al. 2003). Some tissue damages may induce the production of RANTES leading to its increase in plasma. However, to the best of our knowledge, the present report may be the first one showing that neurons can produce RANTES, which affects its plasma levels after ischemic stroke. A future study using neuron-specific RANTES knockout mice would clarify how much neuron-derived RANTES participates in its increase in plasma after ischemic stroke.

\section{2. RANTES is strongly associated with the neuroprotective molecules}




\section{$B D N F, E G F$, and VEGF}

We found that there was a very strong correlation between RANTES and the neurotrophic molecules, BDNF, VEGF, and EGF in plasma after ischemic stroke. These results suggested that brain ischemia may stimulate the production of these molecules via a similar mechanism. Alternatively, gene expression of these molecules may be regulated mutually.

In this context, we found that RANTES directly increased the expression of BDNF, EGF, and VEGF in neuronal cells. Because RANTES was upregulated rapidly in peri-infarct areas after brain ischemia, it may induce the expression of these neuroprotective molecules in nearby neuronal cells. Thus, RANTES may bring about a neuroprotective effect in part by inducing the production of neuroprotective molecules in the brain.

\subsection{Is RANTES good or bad for ischemic stroke?}

It has been reported that infarct volume is significantly smaller in RANTES deficient mice when compared with controls (Terao et al., 2008). This is consistent with the concept that RANTES is a pro-inflammatory chemokine (Maghazachi et al., 1996), and thus simple administration of RANTES would exaggerate the brain damage in acute ischemic stroke. However, it has been reported recently that infarct volume is rather enlarged in CCR5 deficient mice when compared with littermates (Sorce et al., 2010). Based on these reports and our present data, RANTES may be able to play a neuroprotective role via CCR5 
(and CCR3) expressed in neurons in the early phase of ischemic stroke, while it may worsen neuronal damage by breaking the blood-brain barrier (BBB) and inducing invasion of inflammatory cells through CCR1 expressed in vascular cells and white blood cells. The overall role of RANTES in ischemic stroke can be determined by its action on CCR1, CCR3 and CCR5 expressed in different cell types. We speculate that CCR1-mediated BBB breakdown and white blood cell recruitment leading to inflammation surpass CCR5- and (CCR3-) mediated neuroprotection particularly in acute ischemic stroke. Thus, RANTES may work detrimentally during acute ischemic stroke. We speculate that the specific inhibition of RATNES receptors expressed on endothelial cells and white blood cells would bring about a beneficial effect in the acute phase of ischemic stroke.

It is well known that VEGF has a similar function to RANTES. VEGF is produced by neurons in response to hypoxia and can play a neuroprotective role, while it also elicits endothelial growth and migration, thereby causing BBB breakdown (Sun et al., 2003). Thus, VEGF functions detrimentally in the acute phase of ischemic stroke despite its neuroprotective and angiogenic properties. Because RANTES correlated with VEGF, these two molecules may function cooperatively in the acute phase of ischemic stroke.

In conclusion, RANTES is an interesting chemokine where expression is induced drastically in neurons in peri-infarct areas immediately after stroke onset. RANTES may have the potential to protect neurons in an autocrine/paracrine 
manner via neuron-specific receptors. Therefore, selective activation and/or inhibition of chemokine receptors may be therapeutic targets for ischemic stroke in the future.

\section{Experimental procedure}

\section{1. Blood biomarker study for ischemic stroke}

Patients were recruited from the Fukuoka Stroke Registry, a multicenter observational study for acute ischemic stroke in Japan (Kamouchi et al., 2011; Kumai et al., 2012). On admission, the objectives, study design, risks, and benefits were explained in detail to each patient or surrogate family members and written informed consent was obtained. Patients who consented to the study were prospectively enrolled. Patients with ischemic stroke hospitalized within 24 $\mathrm{h}$ after onset and with definite diagnosis by brain imaging were included.

We recruited 171 patients with ischemic stroke who were hospitalized at Kyushu University Hospital, National Hospital Organization Kyushu Medical Center or St. Mary's Hospital in Japan. An equal number of age- and gendermatched controls without a history of cardiovascular disease, such as stroke, coronary heart disease, and atrial fibrillation, were enrolled as healthy subjects from the participants in the Hisayama Study, a worldwide well-known cohort study, in Japan (Kubo et al., 2007; Tanizaki et al., 2000).

\section{2. Measurement of blood biomarkers}


Blood samples were collected from patients at days $0,3,7,14$, and 90 after stroke onset. For healthy subjects, blood samples were obtained at enrollment. Plasma samples were stocked at $-80^{\circ} \mathrm{C}$ until ready for use. We examined plasma concentrations of more than 100 molecules, including BDNF, EGF, VEGF, RANTES and other chemokines, by multiplexed immunoassay (HumanMAP® v 1.6, Rules-Based Medicine, Inc., TX, USA).

Mouse plasma RANTES was measured by an ELISA system (Abcam \#ab100739, Cambridge, UK) according to instructions. Mouse blood was collected in Microtainer ${ }^{\circledR}$ tube with $\mathrm{K}_{2}$-EDTA (BD \#365974, Franklin Lakes, NJ) transcardially from MCAO mice at day 1 after MCAO $(n=8)$ and sham-operated mice $(n=6)$.

\section{3. Experimental MCA occlusion stroke model}

Animal experiments were approved by the animal ethics committee of Kyushu University. We used male C57BI/6J mice aged 7-8 weeks weighing 25-30g. We induced focal cerebral infarction by occlusion of the right middle cerebral artery (MCAO) by endovascular suture as described previously (Shichita et al., 2009). Briefly, the mice were anesthetized with $2 \%(\mathrm{v} / \mathrm{v})$ halothane in a mixture of $70 \%$ $(\mathrm{v} / \mathrm{v})$ nitrous oxide and $30 \%(\mathrm{v} / \mathrm{v})$ oxygen and anesthesia was maintained at $0.8-1 \%$. We inserted a rectal probe and maintained body temperatures between 36.0-37. $0^{\circ} \mathrm{C}$ with a warming lamp. We measured cerebral blood flow (CBF) before and during ischemia using the laser Doppler flowmeter (PeriFlux System 
5000, PERIMED, Stockholm, Sweden) at the ipsilateral parietal bone ( $2 \mathrm{~mm}$ posterior and $5 \mathrm{~mm}$ lateral to bregma). After ligation of the right common carotid artery (CCA), the right middle cerebral artery (MCA) was occluded with a 7-0 nylon monofilament (1696G, Ethicon, Somerville, NJ) coated with a mixture of silicone resin (Xantopren L blue; Heraeus Kulzer, Germany) and a hardener (Activator universal Liquid; Heraeus Kulzer). We inserted an 11-mm long filament from the right CCA. During right MCA occlusion, a reduction in CBF of more than $80 \%$ was confirmed using the laser Doppler flowmeter.

\section{4. Immunohistochemistry and immunofluorescence}

Operated mice were killed with deep anesthesia at day 1 after MCAO and perfused transcardially with ice-cold heparinized saline. The brains were quickly removed, and fresh coronal sections ( $1 \mathrm{~mm}$ thick) were fixed with $4 \%(\mathrm{w} / \mathrm{v})$ paraformaldehyde in phosphate buffered saline (PBS) for immunohistochemistry. Paraformaldehyde-fixed coronal sections were embedded in paraffin sections and cut into $4 \mu \mathrm{m}$ thick slices. Sections were deparaffinized and antigen retrieval was performed. For immunohistochemistry, we inactivated endogenous peroxidase with $0.3 \%(\mathrm{v} / \mathrm{v}) \mathrm{H}_{2} \mathrm{O}_{2}$ in methanol for 30 min at room temperature. The sections were blocked with PBS containing 5\% (v/v) bovine serum albumin and $5 \%(\mathrm{w} / \mathrm{v})$ skim milk for 1 hour at room temperature. The sections were incubated with primary antibodies [RANTES Antibody (AF478, 1:10 dilution, R\&D SYSTEMS, Minneapolis, MN), NeuN (MAB377, 1:50 dilution, MILLIPORE, 
MA), lectin (FL-1171,1:100 dilution, Vector Laboratories, Burlingame, CA), CCR1 (ab1681, 1:200 dilution, abcam), CCR3 (ab36827, 1: 300, abcam), CCR5 (ab1673, 1: 300, abcam)] overnight at $4{ }^{\circ} \mathrm{C}$. For immunoperoxidase staining, slides were washed, and incubated with biotinylated, affinity-purified anti-rabbit or goat immunoglobulin G (Nichirei Corporation, Japan) as a secondary antibody for 30 minutes at room temperature. After avidin-biotin amplification, the slides were washed, and incubated with 3',3'-diaminobenzidine. The slides were counterstained with hematoxylin to visualize the nuclei. For immunofluorescent double labeling, sections were incubated with each primary antibody followed by incubation with the fluorescence-labeled secondary antibody (Invitrogen). Slides were washed and mounted with Vectashield mounting medium containing 4',6-diamidino-2-phenylindole (DAPI) (Vector Laboratories) for nuclear staining. The slides were observed on a BIOREVO BZ-9000 fluorescence microscope (Keyence Corporation, Japan) (Arimura et al., 2012).

\section{5. Cell culture}

Rat pheochromocytoma PC12 cells were used as a neuronal cell model (Ishitsuka et al., 2012). NGF (nerve growth factor: $50 \mathrm{ng} / \mathrm{mL}$ )-treated PC12 cells were plated on collagen type IV-coated dishes (Iwaki Glass, Japan) and were cultured in RPMI1640 (Invitrogen, Carlsbad, CA, USA) supplemented with 10\% (v/v) fetal bovine serum (Invitrogen). Cells were cultured at $37^{\circ} \mathrm{C}$ in $5 \% \mathrm{CO}_{2}$ in a humidified incubator and were treated with RANTES $(50 \mathrm{ng} / \mathrm{mL})$ and the 
indicated inhibitors (50 ng/mL) after serum depletion for $24 \mathrm{~h}$. RANTES was purchased from R\&D SYSTEMS (Recombinant Human CCL5/RANTES, Minneapolis, MN). U0126 (an inhibitor of MEK1) and LY294002 (an inhibitor of PI3K) were purchased from Cell Signaling Technology (CST, Boston, MA, USA).

\section{6. Quantitative PCR}

Total RNA was prepared from cultured cells or mouse organs, such as the brain, liver, and bone marrow, using TRIzol reagent (Invitrogen). One $\mu g$ of total RNA was reverse-transcribed with ReverTra Ace qPCR RT kit (Toyobo, Japan). Using the reverse transcription product as a template, PCR was performed with primers specific for target genes. After preincubation at $94^{\circ} \mathrm{C}$ for $5 \mathrm{~min}, \mathrm{PCR}$ was performed using 30 cycles of denaturation at $94^{\circ} \mathrm{C}$ for $30 \mathrm{~s}$, annealing at $55^{\circ} \mathrm{C}$ for $30 \mathrm{~s}$, and elongation at $72^{\circ} \mathrm{C}$ for $1 \mathrm{~min}$. Quantitative PCR was performed using the LightCycler (Roche, Germany). The RT products $(2 \mu \mathrm{L})$ were amplified in a reaction mixture $(20 \mu \mathrm{L})$ containing $10 \mu \mathrm{L}$ of Thunderbird SYBR qPCR mix (Toyobo) and $0.25 \mu \mathrm{mol} / \mathrm{L}$ of each primer. The mRNA copy numbers were standardized by 18 s ribosomal RNA (18s rRNA) as an internal control (Nakamura et al., 2008). The following primers (Sigma-Aldrich, Japan) were used: mouse RANTES (forward, 5'-ATATGGCTCGGACACCACTC-3'; reverse, 5'-TGACAAAGACGACTGCAAGG-3'), rat RANTES (forward, 5'-ATATGGCTCGGACACCACTC-3'; reverse, 5'-CCACTTCTTCTCTGGGTTGG-3'), rat VEGF-A (forward, 
5'-GCCCATGAAGTGGTGAAGTT-3'; reverse,

5'-ACTCCAGGGCTTCATCATTG-3'), rat BDNF (forward,

5'-TTGTACACTTCCCGGGTGAT-3'; reverse,

5'-GCAGTCTTTTTTATCTGCCGC-3'), rat EGF(forward,

5'-ACACCGAAGGTGGCTATGTC-3'; reverse,

5'-TAGAGTCAGGGCAAGGCAGT-3'), 18s rRNA (forward,

5'-AAACGGCTACCACATCCAAG-3'; reverse,

5'-CCTCCAATGGATCCTCGTTA-3').

\section{7. Immunoblot analyses}

Cultured cells were homogenized in RIPA lysis buffer $(50 \mathrm{mmol} / \mathrm{L}$ Tris- $\mathrm{HCl}, \mathrm{pH}$ 7.5, $150 \mathrm{mmol} / / \mathrm{NaCl}, 1 \% \mathrm{NP}-40,0.5 \%$ deoxycholic acid, $0.1 \%$ SDS, $5 \mathrm{mmol} / \mathrm{L}$ EDTA, $10 \mathrm{mmol} / \mathrm{L} \mathrm{Na} 4 \mathrm{P}_{2} \mathrm{O}_{7}, 0.1 \mathrm{mmol} / \mathrm{L} \mathrm{Na} \mathrm{VO}_{4}, 1 \mathrm{mmol} / \mathrm{L} \mathrm{NaF}$, and protease inhibitors cocktail (Thermo Fisher Scientific, Rockford, IL). Protein concentration was determined using the BCA Protein Assay Kit (Thermo Fisher Scientific). Samples were subjected to SDS-PAGE electrophoresis (20 $\mu \mathrm{g} /$ lane) and transferred onto PVDF membranes. The membranes were incubated for 1 hour with ECL-advance blocking reagent (GE Healthcare, UK) at room temperature, and probed with primary antibodies overnight at $4^{\circ} \mathrm{C}$, washed, and incubated with secondary antibodies (1:100,000 dilution, CST) for 1 hour at room temperature. Blots were developed using the ECL Advance Western Blotting Detection Kit (GE Healthcare) according to manufacturer instructions (Ago et al., 
2008). The primary antibodies used in this study were purchased from Cell Signaling Technology (CST, Boston, MA) [total Akt (\#4691, 1:1,000 dilution), phospho-Akt (Ser473) (\#4060, 1:1,000 dilution), total Erk 1/2 (\#4695, 1:1,000 dilution), phospho-Erk1/2 (Thr202/Tyr204) (\#4370, 1:2,000 dilution), cleaved-caspase 3 (\#9664, 1:200 dilution), total GSK-3 $\beta \quad$ (\#9315, 1:1,000 dilution), phospho- GSK-3 $\beta$ (Ser-9) (\#9336, 1:1,000 dilution)] and Sigma [ $\beta$ -actin (AS441, 1:4,000 dilution)].

\section{8. Cell Viability Assay}

Cells were seeded $1.5 \times 10^{4}$ per well in collagen type IV-coated 96-well plates (BD, Franklin Lakes, NJ) and were treated with U0126 and/or LY294002 after serum depletion for $24 \mathrm{~h}$. One hour later, the cells were treated with recombinant RANTES. Forty-eight hours later, cell viability was assessed using the MTS [3-(4,5- dimethylthiazol-2-yl)-5-(3-carboxymethoxyphenyl)-2-(4sulfophenyl)-2H-tetrazolium, inner salt]-tetrazolium assay (CellTiter 96, Promega, Madison, WI) according to the manufacturer's protocol. Absorbance was measured at $490 \mathrm{~nm}$ (FlexStation 3, Molecular Devices, Sunnyvale, CA).

\section{9. Statistical Analysis}

Statistical analyses between groups were performed using the unpaired Student's $t$ test or one-way ANOVA followed by Bonferroni's post hoc 
comparison test. A value of $p<0.05$ was regarded as statistically significant.

\section{Acknowledgements}

We are grateful to Naoko Kasahara and Eiko Noguchi (Kyushu University) for technical support. The work was supported in part by a Grant-in-Aid for Scientific Research (B) (21390243) from the Ministry of Education, Culture, Sports, Science and Technology, Japan (to T.A.); the Takeda Science Foundation, Japan (to T.A.), the Innovation Center for Medical Redox Navigation (to T.A.); and a Grant-in-Aid for Scientific Research (C) (22590937) from the Ministry of Education, Culture, Sports, Science and Technology, Japan (to M.K.).

\section{Appendix}

REBIOS Investigators

Ryu Matsuo, MD; Noriko Makihara, MD; Kuniyuki Nakamura, MD; Koji Ishitsuka, MD; Asako Nakamura, MD; Koichi Arimura, MD; Ataru Nishimura, MD:

Department of Clinical Science and Medicine, Graduate School of Medical

Sciences, Kyushu University. Yasushi Okada, MD; Masahiro Yasaka, MD, Yoshiyuki Wakugawa, MD: Department of Cerebrovascular Disease and Clinical Research Institute, National Hospital Organization Kyushu Medical Center. Kenji Fukuda, MD; Yoshihisa Fukushima, MD: Department of Cerebrovascular Disease, St. Mary's Hospital. Jun Hata, MD; Seiji Gotoh, MD; Fukuhara: Environmental Medicine, Graduate School of Medical Sciences, Kyushu 
University. Tetsu Isomura, PhD; Teruaki Kobayashi, PhD: Molecuence.

\section{Conflict of Interest}

The authors have no conflicts of interest to declare. 


\section{References}

Ago, T., Liu, T., Zhai, P., Chen, W., Li, H., Molkentin, J.D., Vatner, S.F., Sadoshima, J., 2008. A redox-dependent pathway for regulating class II HDACs and cardiac hypertrophy. Cell. 133, 978-93.

Arimura, K., Ago, T., Kamouchi, M., Nakamura, K., Ishitsuka, K., Kuroda, J., Sugimori, H., Ooboshi, H., Sasaki, T., Kitazono, T., 2012. PDGF receptor beta signaling in pericytes following ischemic brain injury. Curr Neurovasc Res. 9, 1-9.

Cyster, J.G., 1999. Chemokines and cell migration in secondary lymphoid organs. Science. 286, 2098-102.

Daugherty, B.L., Siciliano, S.J., DeMartino, J.A., Malkowitz, L., Sirotina, A., Springer, M.S., 1996. Cloning, expression, and characterization of the human eosinophil eotaxin receptor. J Exp Med. 183, 2349-54.

Ignatov, A., Robert, J., Gregory-Evans, C., Schaller, H.C., 2006. RANTES stimulates $\mathrm{Ca} 2+$ mobilization and inositol trisphosphate (IP3) formation in cells transfected with $\mathrm{G}$ protein-coupled receptor 75. Br J Pharmacol. 149, 490-7.

Ishitsuka, K., Ago, T., Arimura, K., Nakamura, K., Tokami, H., Makihara, N., Kuroda, J., Kamouchi, M., Kitazono, T., 2012. Neurotrophin production in brain pericytes during hypoxia: a role of pericytes for neuroprotection. Microvasc Res. 83, 352-9.

Kamouchi, M., Matsuki, T., Hata, J., Kuwashiro, T., Ago, T., Sambongi, Y., Fukushima, Y., Sugimori, H., Kitazono, T., 2011. Prestroke glycemic control is associated with the functional outcome in acute ischemic stroke: the Fukuoka Stroke Registry. Stroke; a journal of cerebral circulation. 42, 2788-94.

Kubo, M., Hata, J., Ninomiya, T., Matsuda, K., Yonemoto, K., Nakano, T., Matsushita, T., Yamazaki, K., Ohnishi, Y., Saito, S., Kitazono, T., Ibayashi, S., Sueishi, K., lida, M., Nakamura, Y., Kiyohara, Y., 2007. A nonsynonymous SNP in PRKCH (protein kinase $\mathrm{C}$ eta) increases the risk of cerebral infarction. Nat Genet. 39, 212-7.

Kumai, Y., Kamouchi, M., Hata, J., Ago, T., Kitayama, J., Nakane, H., Sugimori, 
H., Kitazono, T., 2012. Proteinuria and clinical outcomes after ischemic stroke. Neurology. 78, 1909-15.

Maghazachi, A.A., Al-Aoukaty, A., Schall, T.J., 1996. CC chemokines induce the generation of killer cells from CD56+ cells. Eur J Immunol. 26, 315-9.

Mirabelli-Badenier, M., Braunersreuther, V., Viviani, G.L., Dallegri, F., Quercioli, A., Veneselli, E., Mach, F., Montecucco, F., 2011. CC and CXC chemokines are pivotal mediators of cerebral injury in ischaemic stroke. Thrombosis and haemostasis. 105, 409-20.

Montecucco, F., Braunersreuther, V., Lenglet, S., Delattre, B.M., Pelli, G., Buatois, V., Guilhot, F., Galan, K., Vuilleumier, N., Ferlin, W., Fischer, N., Vallee, J.P., Kosco-Vilbois, M., Mach, F., 2012. CC chemokine CCL5 plays a central role impacting infarct size and post-infarction heart failure in mice. Eur Heart J. 33, 1964-74.

Murdoch, C., Finn, A., 2000. Chemokine receptors and their role in inflammation and infectious diseases. Blood. 95, 3032-43.

Murphy, P.M., Baggiolini, M., Charo, I.F., Hebert, C.A., Horuk, R., Matsushima, K., Miller, L.H., Oppenheim, J.J., Power, C.A., 2000. International union of pharmacology. XXII. Nomenclature for chemokine receptors. Pharmacol Rev. 52, 145-76.

Nakamura, K., Kamouchi, M., Kitazono, T., Kuroda, J., Matsuo, R., Hagiwara, N., Ishikawa, E., Ooboshi, H., Ibayashi, S., lida, M., 2008. Role of NHE1 in calcium signaling and cell proliferation in human CNS pericytes. Am J Physiol Heart Circ Physiol. 294, H1700-7.

Nomura, S., Uehata, S., Saito, S., Osumi, K., Ozeki, Y., Kimura, Y., 2003. Enzyme immunoassay detection of platelet-derived microparticles and RANTES in acute coronary syndrome. Thromb Haemost. 89, 506-12.

Peng, H., Wen, T.C., Tanaka, J., Maeda, N., Matsuda, S., Desaki, J., Sudo, S., Zhang, B., Sakanaka, M., 1998. Epidermal growth factor protects neuronal cells in vivo and in vitro against transient forebrain ischemiaand free radical-induced injuries. J Cereb Blood Flow Metab. 18, 349-60.

Proudfoot, A.E., Fritchley, S., Borlat, F., Shaw, J.P., Vilbois, F., Zwahlen, C., Trkola, A., Marchant, D., Clapham, P.R., Wells, T.N., 2001. The BBXB motif of RANTES is the principal site for heparin binding and controls 
receptor selectivity. J Biol Chem. 276, 10620-6.

Shichita, T., Sugiyama, Y., Ooboshi, H., Sugimori, H., Nakagawa, R., Takada, I., Iwaki, T., Okada, Y., lida, M., Cua, D.J., Iwakura, Y., Yoshimura, A., 2009. Pivotal role of cerebral interleukin-17-producing gammadeltaT cells in the delayed phase of ischemic brain injury. Nat Med. 15, 946-50.

Slimani, H., Charnaux, N., Mbemba, E., Saffar, L., Vassy, R., Vita, C., Gattegno, L., 2003. Interaction of RANTES with syndecan-1 and syndecan-4 expressed by human primary macrophages. Biochim Biophys Acta. 1617, 80-8.

Song, E.J., Yoo, Y.S., 2011. Nerve growth factor-induced neurite outgrowth is potentiated by stabilization of TrkA receptors. BMB Rep. 44, 182-6.

Sorce, S., Bonnefont, J., Julien, S., Marq-Lin, N., Rodriguez, I., Dubois-Dauphin, M., Krause, K.H., 2010. Increased brain damage after ischaemic stroke in mice lacking the chemokine receptor CCR5. Br J Pharmacol. 160, 311-21.

Struyf, S., Menten, P., Lenaerts, J.P., Put, W., D'Haese, A., De Clercq, E., Schols, D., Proost, P., Van Damme, J., 2001. Diverging binding capacities of natural LD78beta isoforms of macrophage inflammatory protein-1alpha to the CC chemokine receptors 1,3 and 5 affect their anti-HIV-1 activity and chemotactic potencies for neutrophils and eosinophils. Eur J Immunol. 31, 2170-8.

Sun, Y., Jin, K., Xie, L., Childs, J., Mao, X.O., Logvinova, A., Greenberg, D.A., 2003. VEGF-induced neuroprotection, neurogenesis, and angiogenesis after focal cerebral ischemia. J Clin Invest. 111, 1843-51.

Tanizaki, Y., Kiyohara, Y., Kato, I., Iwamoto, H., Nakayama, K., Shinohara, N., Arima, H., Tanaka, K., Ibayashi, S., Fujishima, M., 2000. Incidence and risk factors for subtypes of cerebral infarction in a general population: the Hisayama study. Stroke. 31, 2616-22.

Terao, S., Yilmaz, G., Stokes, K.Y., Russell, J., Ishikawa, M., Kawase, T., Granger, D.N., 2008. Blood cell-derived RANTES mediates cerebral microvascular dysfunction, inflammation, and tissue injury after focal ischemia-reperfusion. Stroke. 39, 2560-70.

Wu, D., 2005. Neuroprotection in experimental stroke with targeted 
neurotrophins. NeuroRx. 2, 120-8.

Yilmaz, G., Granger, D.N., 2010. Leukocyte recruitment and ischemic brain injury. Neuromolecular Med. 12, 193-204.

Zhang, Y., Pardridge, W.M., 2001. Neuroprotection in transient focal brain ischemia after delayed intravenous administration of brain-derived neurotrophic factor conjugated to a blood-brain barrier drug targeting system. Stroke. 32, 1378-84.

Zlotnik, A., Yoshie, O., 2000. Chemokines: a new classification system and their role in immunity. Immunity. 12, 121-7. 


\section{Figure legends}

Figure 1. Plasma concentrations of $\mathrm{C}-\mathrm{C}$ chemokines in 171 stroke patients at day 0 after stroke onset and in age- and gender-matched healthy controls. A, RANTES (CCL5); B, MCP-1 (CCL2); C, MIP-1 $\alpha$ (CCL3); D, MIP-1 $\beta$ (CCL4). Data are shown as the mean \pm standard deviation (SD). E, temporal profile of plasma RANTES from day 0 to 90 after stroke onset is shown. Data are shown as the mean \pm standard error (SE). A value of ${ }^{*} p<0.05$ is regarded as statistically significant against controls. The correlation between RANTES and the neuroprotective molecules, BDNF (F), EGF $(G)$ and VEGF $(H)$, in stroke patients is shown. $r$ denotes the correlation coefficient.

Figure 2. Expression of RANTES in the brain at day 1 after MCAO. Brain infarction made with MCAO was confirmed by hematoxylin-eosin staining (A, B). Plasma RANTES levels in sham-operated $(\mathrm{N}=6)$ and MCAO $(\mathrm{N}=8)$ mice were evaluated by ELISA $\left({ }^{*} p<0.05\right)(C)$. The mRNA expression levels of RANTES in the brain were examined by quantitative PCR. The values were standardized by the expression levels of $18 \mathrm{~s} r \mathrm{RNA}\left(\mathrm{N}=3,{ }^{*} \mathrm{p}<0.05\right)(\mathrm{D})$. Immunohistochemistry shows that RANTES was stained in neuron-like cells strongly in peri-infarct areas (E, F arrows), but not in the contralateral hemisphere $(G)$ and in the brain with sham operation $(\mathrm{H})$ at day 1 after MCAO. Immunofluorescent double-labeling shows that RANTES (red) was stained with NeuN-positive neurons (green) in peri-infarct areas at day 1 after MCAO. 
Figure 3. Expression of the receptors for RANTES (CCR1, CCR3 and CCR5) in the brain at day 1 after MCAO in sham operated mice (A-C), in peri-infarct areas (D-F), and in the contralateral hemisphere (G-I). Immunohistochemistry shows that CCR1 appears to be expressed in vascular cells (D), while CCR3 (E) and CCR5 (F) were expressed in neurons in peri-infarct areas. The expression level of CCR1 $(\mathrm{G})$, CCR3 $(\mathrm{H})$, and CCR5 $(\mathrm{I})$ in the contralateral hemisphere was much lower than that in the peri-infarct areas. Immunofluorescent double-labeling shows that CCR1 was stained with lectin-positive endothelial cells (J), while CCR3 (K) and CCR5 (L) were stained with NeuN-positive neurons in peri-infarct areas at day 1 after MCAO (bar: $20 \mu \mathrm{m})$.

Figure 4. Effects of RANTES on protein activities in the differentiated PC 12 cells. Immunoblot analyses for Akt (A), Erk1/2 (B), GSK-3 $\beta(C)$, and cleaved caspase-3 (D) after treatment with RANTES (50 ng/mL) in PC12 cells. Representative blots and quantitative bar data are shown as the mean \pm SD $(\mathrm{N}=3, * \mathrm{p}<0.05$ vs. $0 \mathrm{~h})$.

Figure 5. Effects of RANTES on mRNA expression of BDNF (A), EGF (B), and VEGF (C) in differentiated PC12 cells. Representative gel images of RT-PCR and quantitative data (mean $\pm \mathrm{SD}(\mathrm{N}=4, * ; \mathrm{p}<0.05))$ are shown. Effects of U0126 or LY29002 on RANTES-mediated expression changes of neurotrophic 
factors (D-F). Quantitative bar graph shows the mean $\pm S D\left(N=4-6,{ }^{*} p<0.05\right.$, NS: not significant).

Figure 6. Effects of RNATES on survival of neuronal cells under serum free conditions. RANTES (50 ng/mL or $500 \mathrm{ng} / \mathrm{mL}$ ) was added (A) with or without U0126 and/or LY294002 (B) to NGF-treated PC12 cells under serum free conditions. Effects of cell survival were assessed using the cell viability assay. Quantitative bar graph shows the mean $\pm S D\left(N=12,{ }^{*} p<0.05,{ }^{* *} p<0.01, N S\right.$ : not significant). 